\title{
Potential role of plasmapheresis in severe cytomegalovirus infection with ongoing immune- mediated hemolysis and low complement level
}

\author{
Sohail Abdul Salim ${ }^{1}$, Yougandhar V. Akula ${ }^{1}$, Bettina B. Noszály ${ }^{2}$, Sabahat Afshan ${ }^{3}$, Mohit Agarwal ${ }^{1}$, \\ Tibor Fülö $\mathbf{p}^{4,5^{*}}$ \\ ${ }^{1}$ Department of Internal Medicine, Division of Nephrology, University of Mississippi Medical Center, Jackson, MS, USA \\ ${ }^{2}$ Kossuth Lajos Preparatory and Demonstration School, University of Debrecen, Hungary \\ ${ }^{3}$ Department of Pediatrics, Division of Pediatric Nephrology, University of Mississippi Medical Center, Jackson, MS, USA \\ ${ }^{4}$ Department of Medicine, Division of Nephrology, Faculty of Medicine, University of Debrecen, Hungary \\ ${ }^{5}$ FMC Extracorporeal Life Support Center, Fresenius Medical Care, Debrecen, Hungary
}

\section{A R T I C L E I N F O}

Article Type:

Case Report

\section{Article History:}

Received: 27 April 2017

Accepted: 13 August 2017

Published online: 8 September 2017

\section{Keywords:}

Complement, Disseminated intravascular coagulopathy, Herpesvirus infection, End-stage renal disease, Myelitis, Plasma exchange, Reactivation, Septic shock

\begin{abstract}
A B S T R A C T
Symptomatic cytomegalovirus (CMV) infections are rare in patients with no previous history of immunosuppressive therapy or condition. A 33-year-old woman with type-2 diabetes, endstage renal disease (ESRD) and unexplained lower extremity weakness was admitted with failure to thrive, ongoing hemolysis, low haptoglobin and C3 levels and suppressed IgM and IgG globulin fractions. Multiple cultures and serologic workup for autoimmune disorders remained negative. CMV viral titer by serum polymerase chain reaction (PCR) revealed massive elevation at 9.2 million copies/mL. Initial CMV IgG titer was positive, IgM titer remained consistently negative and CMV was recovered from cerebrospinal fluid, as well. She received treatment with I.V. ganciclovir, CMV immune globulin (CytoGam) and three sessions of plasmapheresis (PLEX). The extreme viremia without neutropenia, lack of IgM antibody reconversion and CMV-mediated hemolysis with a low complement level were all very unusual features. Timely initiation of PLEX may have contributed to recovery.
\end{abstract}

Implication for health policy/practice/research/medical education:

CMV disease and viremia in the malnourished, chronically ill patients may dramatically differ from those with prior organ transplants. The absence of low white blood cell count or lack of CMV IgM reconversion are not sufficient alone to rule out the clinical diagnosis, only viral DNA testing by PCR. Autoimmune hemolytic anemia with low complement level may occur in some patients with CMV infection which may respond, in addition to specific antiviral therapy, to therapeutic plasma exchange. Please cite this paper as: Salim SA, Akula YV, Noszály BB, Afshan S, Agarwal M, Fülöp T. Potential role of plasmapheresis in severe cytomegalovirus infection with ongoing immune-mediated hemolysis and low complement level. J Renal Inj Prev. 2018;7(3):206-210. doi: 10.15171/jrip.2018.48.

\section{Introduction}

Symptomatic cytomegalovirus (CMV) reactivations are relatively common event in immunosuppressed patients. Typical scenarios would entail severe and prolonged critical illnesses (1-3), organ transplant recipients, advanced human immunodeficiency virus (HIV) infections or otherwise immunosuppressed states. A low white blood cell count is an important clinical clue to the diagnosis in organ transplant recipients. Nonetheless, reports continue to emerge documenting atypical, vasculitis-like presentations of CMV infections in formerly healthy, non-immunosuppressed individuals (4). Other reports are also suggesting that acute kidney injury requiring renal replacement therapy (5) or end-stage renal disease (ESRD) (6) are also predisposing conditions for systemic $\mathrm{CMV}$ reactivation. We report an atypical case of $\mathrm{CMV}$ reactivation in an ESRD patient with multiple systemic complications. 


\section{Case Presentation}

A 33-year old African-American woman presented to the emergency department with mental status changes, syncope and hypotension. For several days prior to admission, she experienced increasing weakness and diarrhea. Her past medical history included type-1 diabetic mellitus with diabetic retinopathy, neuropathy and nephropathy. ESRD was declared four months earlier and she has been receiving maintenance dialysis via a right internal jugular tunneled hemodialysis catheter ever since. She has been experiencing an unexplained bilateral lower extremity weakness and was unable to ambulate since ESRD was declared. She was anemic on arrival (hemoglobin; $6.8 \mathrm{~g} / \mathrm{dL}$ ) and had impaired liver function tests (LFT) (Table 1) as well as low serum albumin for months before presentation (2.6-1.2 g/dL).

Initially, she was thought to be septic but all cultures, including blood, stool and urine returned negative. The physical exam was remarkable for moderate dependent edemas, abdominal distension and profound lower extremity weakness with maintained sensation. Initial differential of elevated LFTs included drug-induced liver injury which failed to improve upon stopping offending agents. She had persisting, occult blood-positive diarrhea but Clostridium difficile testing carried out by polymerase chain reaction (PCR) was negative on three consecutive occasions. Hemolytic work-up revealed hemolytic anemia with thrombocytopenia and elevated lactate dehydrogenase, low haptoglobin level and a mildly positive coombs reaction (Figure 1A-B). Furthermore, she had low $\mathrm{C} 3$ and $\mathrm{CH} 50$ values with low fibrinogen and D-dimer, raising concern for disseminated intravascular coagulation (DIC) (Table 1). However, fibrin degradation products returned within normal limits $(<5 \mu \mathrm{g} / \mathrm{mL}$; $[$ ref.: $<5])$ on day 14 th of the admission. Serum IgG level was relatively low at $930 \mathrm{mg} / \mathrm{dL}$ (reference: 767-1590) while IgM was frankly suppressed at $9 \mathrm{mg} / \mathrm{dL}$ (reference: 40-230). There was no evidence for monoclonal IgG fraction or isolated subclass deficiency. During admission, progressive hypotension emerged, requiring ICU transfer and conversion of renal replacement therapy to slow continuous modality with veno-venous hemofiltration. Extensive evaluations for other sources of infection, including parvovirus, West Nile virus and arbovirus infections, human immunodeficiency virus- 1 and 2 infections (both antibodies and PCR test), rapid plasma reagin test, anti-streptolysin-O and

Table 1. Key biochemical parameters during admission

\begin{tabular}{|c|c|c|c|c|}
\hline & Admission & Day 14 & Day 21 & Day 40 \\
\hline Serum creatinine, $\mathrm{mg} / \mathrm{dL}(0.51-0.95)$ & 3.4 & 3.26 & 1.13 & 2.29 \\
\hline White blood cell count, $10^{3} / \mathrm{mm}^{3}$ & 11.3 & 8.9 & 6.8 & 6 \\
\hline \multicolumn{5}{|l|}{ White blood cell differential,\% } \\
\hline Neutrophyls (44-65) & 86 & 85 & 80 & \\
\hline Lymphocytes (25-46) & 12 & 9 & 16 & \\
\hline Monocytes (1-10) & 1 & 2 & 2 & \\
\hline Eosinophyls (0-9) & 0 & 2 & 2 & \\
\hline Hemoglobin, g/dL & 6.8 & 7.2 & 7.2 & 6.7 \\
\hline Platelets, $10^{3} / \mathrm{mm}^{3}$ & 110 & 50 & 34 & 132 \\
\hline Lactate dehydrogenase, U/L (normal range: 135-214) & & 461 & 206 & 362 \\
\hline Haptoglobin, mg/dL (30-200) & & 0 & 42 & 35 \\
\hline Direct antiglobulin test (Coombs) positivity & & (+) Positive & Negative & \\
\hline Complement 3, mg/dL (90-180) & 61 & 63 & & \\
\hline Complement 4, mg/dL (10-40) & 28 & 20 & & \\
\hline Prothrombin time, International Normalized Ratio & 1.67 & 1.96 & 1.29 & 2.02 \\
\hline Fibrinogen, mg/dL (200-393) & & 36 & 191 & 167 \\
\hline Bilirubin, mg/dL (0-1) & 18 & 12.8 & 9.7 & 11.9 \\
\hline Aspartate aminotransferase, U/L (0-32) & 69 & 61 & 53 & 43 \\
\hline Alanine Aminotransferase, U/L (0-33) & 66 & 118 & 45 & 49 \\
\hline Alkaline phosphatase, U/L (35-104 U/L) & 523 & 118 & 77 & 143 \\
\hline Serum albumin, g/dL (3.5-5.5) & 1.3 & 1.9 & 2 & 2 \\
\hline Procalcitonine, $\mathrm{ng} / \mathrm{mL}(0.00-0.05)$ & 2.95 & $2.64^{* * *}$ & & 2.02 (Day \#38) \\
\hline D-dimer, ng/mL $(0-500)$ & & 1254 & & \\
\hline Thyroid stimulating hormone (0.27-4.20), $\mu \mathrm{IU} / \mathrm{mL}$ & & $3.33^{*}$ & 9.15 & \\
\hline Total T3 (0.80 - 2.0), ng/mL & & $0.21 * *$ & & \\
\hline Free T3 $(200-440), \mathrm{pg} / \mathrm{dL}$ & & $63.4^{* *}$ & & \\
\hline Free T4 (0.93-1.7), ng/dL & & $0.433^{*}$ & 1.07 & \\
\hline Anti-CMV IgG (negative) & Positive & Positive & & \\
\hline Anti-CMV IgM (negative) & Negative & Negative & & \\
\hline CMV PCR titer [plasma], /mL & $9200000 *$ & $1600000 * * *$ & 115000 (Day \#25) & 2600 (Day \#33, 38) \\
\hline
\end{tabular}

*obtained on Day \#9; **obtained on Day \#12; ***obtained on Day \#16.

Day \#41 is the day of discharge. 
tuberculosis interferon release assays all returned with negative or unremarkable results. Serology testing for acute hepatitis-A, B and C, autoimmune hepatitis or systemic lupus erythematosus was also negative. Serum folate, vitamin B12, methylmalonic acid and zinc levels returned within normal limits. Absolute CD4 count was preserved between $644-1042 / \mathrm{mm}^{3}$, with CD4:CD8 ratio 1.14-1.3. While the peripheral blood smear revealed a "moderate" amount of schistocyte formation, the ADAMTS-13 activity was not suppressed. CT scan of the abdomen revealed diffuse wall thickening and edemas in the entire colon without distention, "most suspicious for infectious and noninfectious colitis, including C. difficile/pseudomembranous colitis." A bone marrow biopsy recovered normocellular marrow with tri-lineage hematopoiesis and no evidence of hemophagocytosis. For coagulopathy, she received aggressive support, including packed red blood cells and multiple other blood products (Figure 1C-D). Given the context of chronic unexplained failure to thrive, persistently low albumin, chronic diarrhea and elevated liver enzymes, a CMV virus PCR plasma titer was obtained with markedly abnormal results (9200000 million copies/mL), consistent upon multiple repeats $(2600000-1600000$ copies/mL, both from plasma and serum). We felt that the patient's illness represented a truly life-threatening systemic CMV infection with specific involvement of the colon, esophagus and liver strongly suspected; additionally, CNS involvement was strongly suspected due to the lower extremity weakness the patient experienced upon admission. A CMV-specific therapy was instituted both with I.V. ganciclovir and CMV immune globulin (CytoGam). Additionally, in the context of a profound systemic illness, coagulopathy and hemolysis with low complement levels and favorable results on sporadic reports in the past $(7,8)$, we elected to institute plasma exchange (PLEX) treatments with a centrifugal system, exchanging x1.5 predicted plasma volume with fresh frozen plasma. The clinical picture of septic shock and DIC resolved after three rounds of PLEX. Her subsequent hospital course was complicated by a new onset left facial drop and left eye blindness; lumbar puncture also returned with a CMV-PCR-positive result from the spinal fluid. CNS magnetic resonance imaging revealed parenchymal thickening compatible with both infectious process and meningitis but there was no evident stigmata of a stroke. She gradually improved, including with decline of serum CMV PCR titers (Table 1) and was discharged to a rehabilitation facility on continued maintenance hemodialysis.

\section{Discussion}

CMV can infect multiple cells including endothelial cells, epithelial cells, smooth muscle cells, fibroblasts, leukocytes, as well as retinal and dendritic cells. An intact adaptive immune response to $\mathrm{CMV}$ requires both humoral and cellular immunity which helps in controlling the infection once the virus enters latency and prevents
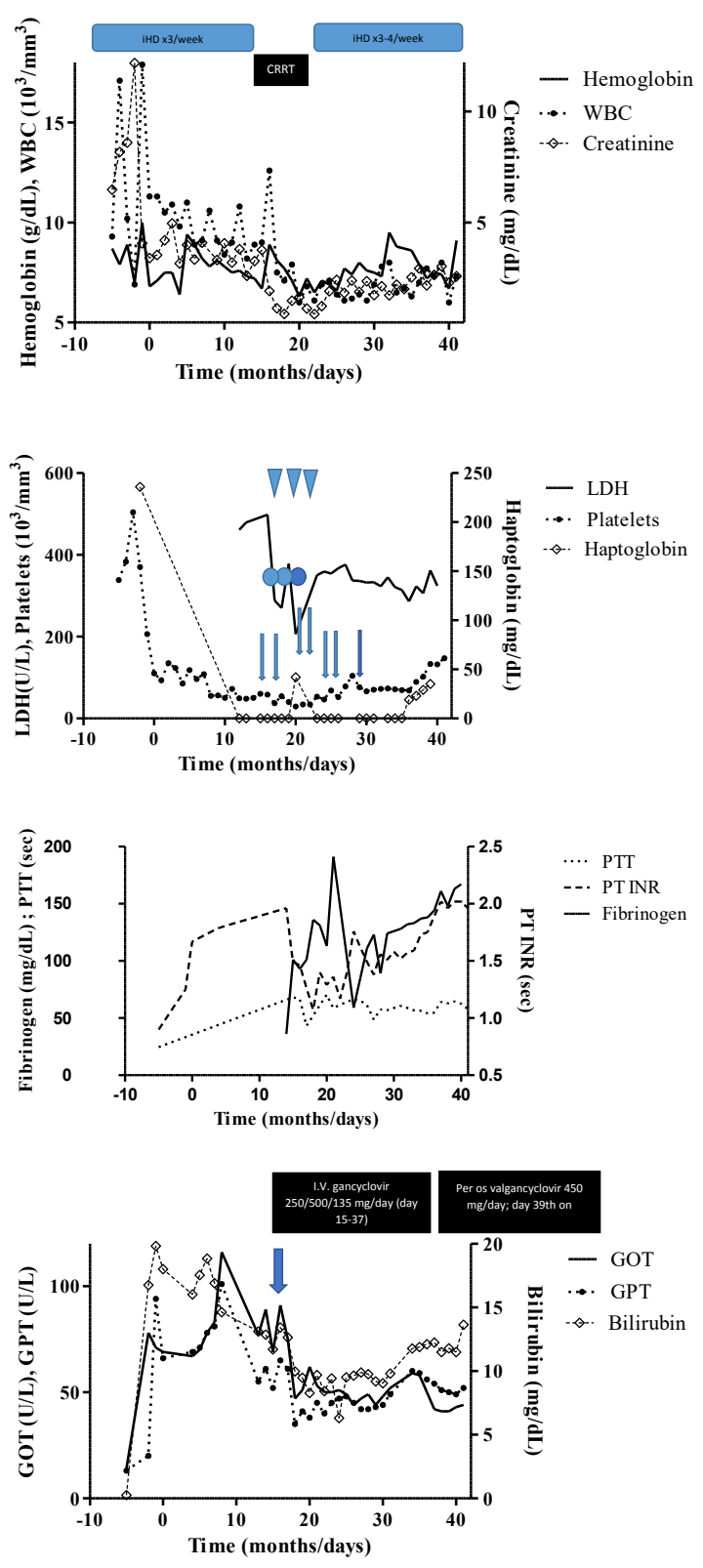

Figures 1 (A-D). "0" point represents index inpatient admission; scale before admission in months, after admission in days. (A) Time-course of serum creatinine, white blood cell (WBC) count and hemoglobin during the admission. Abbreviations: CRRT, continuous renal replacement therapy; iHD, intermittent hemodialysis. (B) Time-course of lactate dehydrogenase $(\mathrm{LDH})$, platelets and haptoglobin during admission. Marks: cryoprecipitate, arrow; fresh frozen plasma, down-pointing triangle; plasma exchange therapy, circle. Plasma exchange commenced on day 17,19 and 21 of the hospitalization. Reference range for LDH: 135-214 Unit/L; haptoglobin: 30-200 $\mathrm{mg} / \mathrm{dL}$. (C) Time-course of (activated) partial thromboplastin time (PTT), prothrombin time International Normalized Ratio (PT INR) and fibrinogen during admission. Reference range for fibrinogen: $200-393 \mathrm{mg} / \mathrm{dL}$. (D) Time-course of glutamic-oxaloacetic transaminase (GOT) [AST, aspartate aminotransferase), glutamic-pyruvic transaminase (GPT) [ALT, alanine aminotransferase] and (total) bilirubin levels during admission. On day 15 arrow mark indicating CMV I.V. IgG (Cytogam) therapy. Discharge on per os valgancyclovir 450 $\mathrm{mg} / \mathrm{d}$. 
reactivation. Whether ESRD can be qualified as an immunosuppressed state is certainly debatable (9) but ESRD causes impairment in both innate and adaptive immune responses. Decreased endocytosis and impaired maturation of monocytes and dendritic cells in the uremic state $(10,11)$, as well as impaired functions of toll-like receptors are demonstrable (12), contributing to increased susceptibility to infections.

First of all, the most atypical feature of our current case was the enormous elevation of the CMV viral titer by PCR, in the absence of qualifying immunosuppressive condition. In fact, this marked elevation of viral PCR was unseen by any us before in our career. She had no history of childhood illnesses, recurrent infections or other stigmata of preexisting immune deficiency before the current illness. The elevated CMV PCR titer was repeatedly demonstrated, both from serum and plasma and declined appropriately upon improvement of the patient's condition. She had a preserved her WBC count, thus her presentation was very different from what we would encounter in solid organ transplant recipients. This preserved WBC count may have contributed to the extremes of viral copy number, as the virus is an intracellular pathogen and multiply in mononuclear cells. PLEX removes all subcellular structures and conceivably some of the virus-antibody immune complexes (initial viral PCR titer in plasma: $9200000 \mathrm{copy} / \mathrm{mL}$ versus serum: $2600000 \mathrm{copy} / \mathrm{mL}$ ), presumably contributing to the decrease of viral load and improvement in the hemolytic processes both. Similarly, the clinical picture of CMV infection-induced septic shock resolved after three rounds of PLEX and with continued slow veno-venous hemofiltration. It should be noted that PLEX alone may have some benefits for the most critically ill subjects (13). While her markedly abnormal thyroid function tests could be ascribed to the critical illness alone (14), we speculate that PLEX - as part of its multi-system support - effectively provided replacement for thyroid hormones as well.

Second, her illness was accompanied by a profound immune-mediated complement activation, low platelet counts and consumptive coagulopathy. In cell models, CMV-infected human umbilical vein endothelial cells are known to develop a pro-coagulant phenotype (15). While red blood cell fragmentation was present on the peripheral smear, our Hematology team doubted hemolytic-uremic syndrome. The positive Coombs reaction was likely representing the absorption of viral particles and immune complexes on the surface of red blood cells. Temporarily, the coagulopathy responded well to the initiation of PLEX and presumably represented the combination of systemic inflammatory reaction and relatively poor liver synthetic function. Only very limited published experience addresses the medical therapy for DIC beyond antiviral and hyper-CMV immunoglobulin. Two of these were published after our index observation in 2016, one utilizing PLEX (7), while another applying antiviral therapy and glucocorticoids alone (16).
Third, there was a lack of detectable IgM during the illness. The presence of pre-existing CMV IgG positivity classified her illness as a CMV reactivation. Unlike in a similar case reported in the past (7), we have not observed $\mathrm{CMV}$-specific immunoglobulin IgM re-conversion during her illness. The underlying cause of immune paralysis remained unclear. Repeated testing for HIV, including by PCR, remained negative and her T-helper (CD4) count was also preserved. She had suppressed IgM IgG and relatively low-end IgM levels, but it is unclear whether these represented an underlying disease or appeared as a consequence of her chronically ill state with suppressed nutrition and ongoing inflammation.

Fourth, she clearly had CNS involvement with the CMV virus. Both transverse myelitis $(17,18)$ and optic nerve impairment caused by CMV (19) has been described before. Unfortunately, in our patient the CNS process progressed and new defects developed while being on CMV-specific therapy. It can be reasonably argued that I.V., foscarnet would have been a better choice for the patient owing to CNS penetration.

Altogether, this case broadens our horizon of CMVassociated illnesses. CMV can infect multiple cells including endothelial cells, epithelial cells, smooth muscle cells, fibroblasts, leukocytes, as well as retinal and dendritic cells. An intact adaptive immune response to $\mathrm{CMV}$ requires both humoral and cellular immunity which helps in controlling the infection once the virus enters latency and prevents reactivation. Whether ESRD can be qualified as an immunosuppressed state is certainly debatable (9) but ESRD causes impairment in both innate and adaptive immune responses. Decreased endocytosis and impaired maturation of monocytes and dendritic cells in the uremic state $(10,11)$, as well as impaired functions of toll-like receptors are demonstrated (12), contributing to increased susceptibility to infections. The underling etiology and unifying diagnosis for her systemic illness remained unclear until the diagnosis secured by viral PCR. In critically ill patients with consumptive coagulopathy, suppressed complement levels or hemolytic anemia, CMV should be strongly considered in the differential. The absence of mononucleosis-type symptoms is not sufficient to rule out the diagnosis on clinical grounds. Our case demonstrated that CMV viral load measurement was more helpful for recognizing systemic CMV reactivation than obtaining CMV-specific IgM antibodies.

\section{Conclusion}

In summary, severe CMV disease should be on the differential of malnourished, chronically ill patients presenting with an unexplained systemic illness.

\section{Acknowledgements}

Parts of this project has been presented in poster format at the American Society of Nephrology 2016 Kidney Week Meeting in Chicago, IL. J Am Soc Nephrol 2016; 27:643A. 


\section{Authors' contribution}

SAS and YVA were Nephrology Fellows at the Department of Medicine, University of Mississippi Medical Center during the date collection and prepublication phase of the article (Class of 2017). SA has participated in the data collection. MA is currently affiliated with University of Wisconsin, Department of Medicine, Division of Nephrology and participated in the care of the patient. TF is a former faculty member of the University of Mississippi Medical Center, currently affiliated with the Medical University of South Carolina and the Ralph H. Anderson VA Medical Center in Charleston, SC and participated in the care of the patient. All authors have participated in preparation and writing of the manuscript. We sincerely appreciated the assistance of Mr. Attila Lénárt-Muszka during editing and grammar review.

\section{Conflicts of interest}

The authors declared no competing interests.

\section{Ethical considerations}

This paper has been prepared in accord with the requirements of the HIPAA privacy regulations and the Helsinki Declaration. As per the policies of the University of Mississippi Medical Center's Institutional Review Board (IRB), case reports do not need IRB approval. A written permission for scientific publication has been obtained from the patient discussed herein.

\section{Funding/Support}

None.

\section{References}

1. Jaber S, Chanques G, Borry J, Souche B, Verdier R, Perrigault $\mathrm{PF}$, et al. Cytomegalovirus infection in critically ill patients: associated factors and consequences. Chest. 2005;127:23341. doi: 10.1378/chest.127.1.233.

2. von Muller L, Klemm A, Weiss M, Schneider M, SugerWiedeck H, Durmus N, et al. Active cytomegalovirus infection in patients with septic shock. Emerg Infect Dis. 2006;12:1517-22. doi: 10.3201/eid1210.060411.

3. Kalil AC, Florescu DF. Prevalence and mortality associated with cytomegalovirus infection in nonimmunosuppressed patients in the intensive care unit. Crit Care Med. 2009;37:2350-8. doi: 10.1097/CCM.0b013e3181a3aa43.

4. Chiang TT, Lin TC, Wu RX, Chiu CC, Yang YS. Recurrence of Cytomegalovirus Viremia Causing Gastrointestinal Tract Bleeding at Different Sites in an Immuno-competent Patient. Am J Med Sci. 2017;353:607-9. doi: 10.1016/j. amjms.2016.11.008.

5. Farah Musa AR, Fulop T, Kokko K, Kanyicska B, Lewin JR, Csongradi E. Cytomegalovirus colitis in a critically ill, dialysis-dependent, acute kidney injury patient without immunosuppressive therapy. Clin Nephrol. 2015;84:44-9. doi: $10.5414 / \mathrm{cn} 108417$.
6. Kim SH, Kim YS, Kim HW, Yoon HE, Kim HK, Kim YO, et al. A case of cytomegalovirus colitis in an immunocompetent hemodialysis patient. Hemodial Int. 2011;15:297-300. doi: 10.1111/j.1542-4758.2010.00520.x.

7. Niewold TB, Bundrick JB. Disseminated intravascular coagulation due to cytomegalovirus infection in an immunocompetent adult treated with plasma exchange. Am J Hematol. 2006;81:454-7. doi: 10.1002/ajh.20602.

8. Muller NF, Schampera M, Jahn G, Malek NP, Berg CP, Hamprecht K. Case report: severe cytomegalovirus primary infection in an immunocompetent adult with disseminated intravascular coagulation treated with valganciclovir. BMC Infect Dis. 2016;16:19. doi: 10.1186/s12879-016-1343-3.

9. Anding K, Gross P, Rost JM, Allgaier D, Jacobs E. The influence of uraemia and haemodialysis on neutrophil phagocytosis and antimicrobial killing. Nephrol Dial Transplant. 2003;18:2067-73. doi: 10.1093/ndt/gfg330.

10. Lim WH, Kireta S, Leedham E, Russ GR, Coates PT. Uremia impairs monocyte and monocyte-derived dendritic cell function in hemodialysis patients. Kidney Int. 2007;72:1138-48. doi: 10.1038/sj.ki.5002425.

11. Verkade MA, van Druningen CJ, Vaessen LM, Hesselink DA, Weimar W, Betjes MG. Functional impairment of monocyte-derived dendritic cells in patients with severe chronic kidney disease. Nephrol Dial Transplant. 2007;22:128-38. doi: 10.1093/ndt/gfl519.

12. Ando M, Shibuya A, Tsuchiya K, Akiba T, Nitta K. Reduced expression of Toll-like receptor 4 contributes to impaired cytokine response of monocytes in uremic patients. Kidney Int. 2006;70:358-62. doi: 10.1038/sj.ki.5001548.

13. Nguyen TC, Kiss JE, Goldman JR, Carcillo JA. The role of plasmapheresis in critical illness. Crit Care Clin. 2012;28(3):453-68, vii. doi: 10.1016/j.ccc.2012.04.009.

14. Kumar E, McCurdy MT, Koch CA, Hamadah A, Fülöp T, Gharaibeh KA. Functional impairment of thyroid function in critically ill patients in the Intensive Care Units. Am J Med Sci. 2017. doi: 10.1016/j.amjms.2017.06.026.

15. van Dam-Mieras MC, Muller AD, van Hinsbergh VW, Mullers WJ, Bomans PH, Bruggeman CA. The procoagulant response of cytomegalovirus infected endothelial cells. Thromb Haemost. 1992;68:364-70.

16. Chagla Z, Siegal D, El-Helou P, Lim W, Rudkowski J. Severe Acute Cytomegalovirus Infection Complicated by Disseminated Intravascular Coagulation and Pneumonitis in a Healthy Female. J Gen Intern Med. 2016;10:36-38.

17. Giobbia M, Carniato A, Scotton PG, Marchiori GC, Vaglia A. Cytomegalovirus-associated transverse myelitis in a non-immunocompromised patient. Infection. 1999;27:22830.

18. Arslan F, Yilmaz M, Paksoy Y, Karagoz E, Mert A. Cytomegalovirus-associated transverse myelitis: a review of nine well-documented cases. Infect Dis (Lond). 2015;47:712. doi: 10.3109/00365548.2014.964763.

19. Baglivo E, Leuenberger PM, Krause KH. Presumed bilateral cytomegalovirus-induced optic neuropathy in an immunocompetent person. A case report. J Neuroophthalmol. 1996;16:14-7.

Copyright $\odot 2018$ The Author(s); Published by Nickan Research Institute. This is an open-access article distributed under the terms of the Creative Commons Attribution License (http://creativecommons.org/licenses/by/4.0), which permits unrestricted use, distribution, and reproduction in any medium, provided the original work is properly cited. 